J.-H. Baumert

B. Spellerberg

\title{
Mikrobiologisches Monitoring und kalkulierte Antibiotikatherapie beim akuten Lungenversagen
}

\section{Microbiological monitoring and calculated antibiotic therapy in acute pulmonary failure}

Summary ARDS may be the cause as well as a consequence of pulmonary infection. More than two thirds of patients exhibit systemic infection or sepsis which may also have an extrapulmonary

Eingegangen: 31. Juli 2002

Akzeptiert: 13. August 2002

\section{Serie:}

Die Intensivtherapie

bei akutem Lungenversagen

Herausgegeben von R. Rossaint und R. Kuhlen, Aachen

\section{J.-H. Baumert (}

Klinik für Anästhesiologie

Universitätsklinikum Aachen

Pauwelsstr. 30

52057 Aachen, Germany

\section{B. Spellerberg}

Institut für Medizinische Mikrobiologie

Universitätsklinikum Aachen

aktuelle Adresse:

Abteilung für Medizinische Mikrobiologie und Hygiene

Universitätsklinikum Ulm

Robert-Koch-Str. 8

89081 Ulm, Germany cause. As relevant microorganisms from the respiratory tract are isolated in only half of the patients and because of the acuteness and severity of the disease, the initial treatment often has to be a calculated antibiotic therapy. This should cover the microbiological spectrum associated with the initial disease. Therefore, it has to be different for community-acquired or ventilator-associated pneumonia as well as primary sepsis or extrapulmonary infection.

Respiratory tract and/or blood specimens for microbiological diagnostics should be collected prior to antibiotic treatment in all cases. In complicated cases and in immunosuppressed patients, viral (mainly CMV) as well as fungal infections have to be taken into account. The indication for calculated antibiotic therapy in patients without clinical signs of infection (post-traumatic or transfusion-induced ARDS) is still debated.

Key words Acute respiratory distress syndrome (ARDS) pneumonia - infection antibiotic

\section{Zusammenfassung Das ARDS} kann sowohl Grundlage als auch Folge einer pulmonalen Infektion sein. Bei mehr als zwei Drittel der Patienten liegt eine systemische Infektion oder Sepsis vor, die auch einen extrapulmonalen Ausgangspunkt haben kann. Da nur in der Hälfte der Fälle ein relevanter Erreger aus dem Respirationstrakt isoliert werden kann und es sich um ein akutes, schweres Krankheitsbild handelt, muss primär meist eine kalkulierte antibiotische Therapie eingeleitet werden. Diese sollte das nach der Genese der Erkrankung wahrscheinliche Erregerspektrum vollständig erfassen. Hierbei muss nach ambulant erworbener bzw. beatmungsassoziierter Pneumonie oder primärer Sepsis und gegebenenfalls extrapulmonaler Infektion unterschieden werden. In jedem Fall sollten vor Beginn der Behandlung Material aus dem Respirationstrakt und/oder Blutkulturen zur mikrobiologischen Diagnostik gewonnen werden. Im Verlauf komplizierter Fälle bzw. bei immunsupprimierten Patienten müssen auch virale (vor allem CMV) und Pilzinfektionen berücksichtigt werden. Ob beim ARDS ohne klinische Infektionszeichen (posttraumatische oder transfusionsinduzierte Formen) eine kalkulierte antibiotische Therapie indiziert ist, ist weiterhin nicht geklärt.

\section{Schlüsselwörter}

Acute respiratory distress syndrome (ARDS) - Pneumonie Infektion - Antibiose 


\section{ARDS und pulmonale Infektion}

Der akute Beginn einer respiratorischen Insuffizienz $\left(\mathrm{PaO}_{2} / \mathrm{FiO}_{2}\right.$-Quotient $\left.<300 \mathrm{mmHg}\right)$ mit bilateralen Infiltraten im Röntgenbild bei normaler linksventrikulärer Funktion (linker Vorhofdruck $<18 \mathrm{mmHg}$ ) wird nach der Definition der Konsensus-Konferenz als „akute Lungenschädigung" (ALI) beschrieben; deren schwerste Form $\left(\mathrm{PaO}_{2} / \mathrm{FiO}_{2}\right.$-Quotient $<200$ $\mathrm{mmHg}$ ) erhält danach die Bezeichnung „Acute Respiratory Distress Syndrome" (ARDS) (6). Sowohl ALI als auch ARDS weisen Symptome einer Infektion auf. Dazu gehören Fieber, Leukozytose sowie die genannten pulmonalen Infiltrate. Neben einer Pneumonie können jedoch verschiedene andere Erkrankungen Ursache eines ARDS sein. Tabelle 1 gibt einen Überblick über die Häufigkeitsverteilung.

Die Abgrenzung der anderen möglichen Ursachen des ARDS von einer Pneumonie ist jedoch schwierig (41). Es gelingt bei etwa der Hälfte der Patienten mit ARDS, den Erreger einer pulmonalen Infektion nachzuweisen; darin stimmen alle größeren Untersuchungen überein $(15,27)$. Allerdings wird die Korrelation von mikrobiologischen Befunden mit klinischen Zeichen der Pneumonie als schlecht beschrieben (49). Dem gegenüber fand sich aber bei $70 \%$ einer Gruppe verstorbener Patienten mit ARDS histologisch eine Pneumonie. In einer anderen Untersuchung an Lungenbiopsien von ARDS-Patienten ohne mikrobiologischen Befund wurde in $41 \%$ der Fälle eine Fibrose und bei der Mehrzahl der übrigen eine CMV-Infektion nachgewiesen; eine bakterielle Infektion lag dagegen nur bei etwa $14 \%$ vor (41).

In einer Untersuchung von Headley und Mitarbeitern an 43 Patienten, die mit der Diagnose ARDS aufgenommen wurden, ließen sich zwei Gruppen unterscheiden: Von den 25 Patienten mit primärem Lungenschaden hatten 19 eine Pneumonie, bei 6 lag ursächlich eine Aspiration von Mageninhalt oder eine toxische Genese vor. Ein sekundäres Lungenversagen, d.h. als Folge einer extrapulmonalen Grunderkrankung, ging bei 7 Patienten auf eine extrapul- monale Infektion, bei 3 auf eine primäre Sepsis und bei den übrigen 8 auf Trauma, Transfusionsreaktion etc. zurück (24). Im Verlauf hatten fast $70 \%$ der Patienten eine Sepsis (s. Tab. 1). Diese Zahl belegt, dass Infektionen im Zusammenhang mit ARDS auch dann häufig sind, wenn die primäre Ursache des Lungenversagens nicht eine Pneumonie ist.

Die klinische Situation von Patienten mit ADRS ist daher geprägt durch Symptome einer akuten Lungeninfektion mit der Schwierigkeit, einen relevanten pulmonalen Erreger zu identifizieren, sowie die häufige Assoziation mit primärer Sepsis und extrapulmonalen Infektionen. Dies führt dazu, dass der Intensivmediziner in der Behandlung des ARDS überwiegend auf eine kalkulierte antibiotische Therapie angewiesen ist. Für deren Konzeption ist die Genese des Lungenversagens von Bedeutung, da die ursächliche Erkrankung das in Frage kommende Erregerspektrum bestimmt.

\section{Genese des ARDS und Konsequenzen für Diagnostik und Therapie}

\section{Ambulant erworbene Pneumonie („Community Acquired Pneumonia“)}

Bei Patienten mit ambulant erworbener Pneumonie führen die typischen Symptome wie Schwäche, Fieber, Husten, atemabhängige thorakale Schmerzen sowie schließlich Atemnot zur Aufnahme im Krankenhaus. Auch bei Patienten, die nach einem Trauma eine „frühe" Pneumonie (innerhalb der ersten $48 \mathrm{~h}$ ) entwickeln (45), liegt in der Regel eine ambulant erworbene Pneumonie vor. Antonelli konnte für diese frühe Pneumonie einen protektiven Effekt einer frühzeitigen Intubation und Beatmung nachweisen (wahrscheinlich durch Schutz vor Mikroaspiration von Mund-/Rachenflora), während eine fünf Tage oder länger dauernde Beatmung der primäre Risikofaktor für eine „späte“, d.h. nosokomiale Pneumonie

Tab. 1 Ursachen des ARDS

\begin{tabular}{lllccc}
\hline Autor & Trauma & Pneumonie & Sepsis (primär) & Aspiration & Andere \\
\hline $\begin{array}{l}\text { Lewandowski } \\
1997(n=122)\end{array}$ & 36 & 32 & 11,5 & 9 & 11,5 \\
$\begin{array}{l}\text { Headley } \\
1997(n=43)\end{array}$ & 4,7 & 44 & 7 & 11,6 & 32,7 \\
Eisner & 10,6 & 35,5 & $(67 \%$ gesamt $)$ & & 12,8 \\
$2001(n=902)$ & & & 26,2 & 14,9 & \\
\hline
\end{tabular}

Verteilung der ARDS-Ursachen (in \%) in der operativen (oben) und konservativen Intensivmedizin (Mitte) (24) sowie in der Datenbasis des ARDS Network (unten) (Eisner MD, Thompson T et al. (2001) Am J Respir Crit Care Med 164:231-236). In der Untersuchung von Headley et al. sind unter "andere“ überwiegend (7 Patienten) extrapulmonale Infektionen eingeschlossen. Insgesamt hatten 67\% der Patienten eine Sepsis (24) 
war (s.u.) (1). Aktuelle prospektive Studien belegen jedoch den Nutzen der nichtinvasiven Beatmung bei Patienten mit Pneumonie und akuter respiratorischer Insuffizienz. Confalonieri und Mitarbeiter erreichten bei 56 Patienten mit ARDS als Folge einer ambulant erworbenen Pneumonie in der Gruppe mit nichtinvasiver Beatmung eine Reduktion der Intubationen sowie der Dauer der Intensivtherapie. Bei Patienten mit COPD gelang mit diesem Verfahren sogar eine Verbesserung der 2-Monats-Überlebensrate (13). Bei 52 immunsupprimierten Patienten mit Pneumonie und ARDS erreichten Hilbert et al. signifikante Reduktionen der Intensiv- und Krankenhausletalität durch nichtinvasive Beatmung (25).

\section{Mikrobiologische Diagnostik}

Die klinische Diagnose einer ambulant erworbenen Pneumonie bei einem ARDS-Patienten macht eine umfangreiche mikrobiologische Diagnostik erforderlich. Die diagnostischen Maßnahmen sollten neben den bakteriellen Erregern einer typischen Pneumonie die Erreger atypischer Pneumonien, Viren und vor allem bei immunsupprimierten Patienten Pilze erfassen. Es sollte in jedem Fall angestrebt werden, vor Einleitung einer empirischen Antibiotikatherapie Material aus dem Respirationstrakt zur Diagnosesicherung zu gewinnen, da nach Einleitung der Antibiose mit einem erheblichen Verlust der diagnostischen Sensitivität zu rechnen ist. Bei beatmeten Patienten wird dies in der Regel Trachealsekret (TS) sein. Bronchoalveoläre Lavage (BAL) und Bürstenbiopsie weisen in einigen Untersuchungen eine bessere Sensitivität und Spezifität auf, bergen aber auch das nicht unerhebliche Risiko einer Bronchoskopie beim beatmeten Patienten. Ein Vergleich zahlreicher Studien $\mathrm{zu}$ invasiven (BAL/ Bürste) und nicht invasiven Techniken (TS) der Materialgewinnung aus dem Respirationstrakt ergab nur geringe Unterschiede zwischen den einzelnen Techniken (4), so dass es gerechtfertigt erscheint, invasive Techniken vor allem bei Patienten mit diagnostischen Problemen einzusetzen (35). Die mikrobiologische Untersuchung von Material, das mittels BAL oder Bürste gewonnen wurde, sollte quantitativ erfolgen (36), wobei in der Regel Erregermengen von $\geq 10^{4} / \mathrm{ml}$ gut mit dem histologischen bzw. mikrobiologischen Nachweis einer Pneumonie aus Lungengewebsproben korrelieren. Feinnadelpunktionen oder offene Thorakotomien aus diagnostischen Gründen sind sicherlich als ultima ratio nur in seltenen Fällen indiziert. Die Technik der transtrachealen Aspiration ermöglicht an sich eine kontaminationsfreie Materialgewinnung, hat sich jedoch weder in den USA noch in Europa in größerem Umfang etablieren können.

Die Anfertigung eines Grampräparates aus den gewonnenen Respirationstraktmaterialien erlaubt ei- ne Beurteilung der Qualität des gewonnenen Materials. Bronchial- bzw. Trachealsekret sollte weniger als 10 Plattenepithelzellen und mehr als 25 Granulozyten pro Gesichtsfeld aufweisen, höhere Plattenepithelzellmengen weisen auf eine Kontamination mit Sekret aus dem oberen Respirationstrakt hin. Invasiv mittels BAL oder geschützter Bürste gewonnenes Material sollte $<1 \%$ Plattenepithelien enthalten. Die Bedeutung des Keimnachweises im initialen Grampräparat wird in der internationalen Literatur kontrovers beurteilt. Bei eindeutigem Nachweis von Bakterien im Präparat sollte die eingeleitete Antibiose diese natürlich auf jeden Fall erfassen. Da jedoch mehrfach über eine schlechte Korrelation zwischen dem Ergebnis der Gramfärbung und dem endgültigen Kulturergebnis berichtet wurde $(1,39)$, sollte die Umstellung der empirisch eingeleiteten Antibiose auf eine gezielte Antibiotikatherapie erst nach Vorliegen des Kulturresultates erfolgen.

Bei jedem stationär wegen einer Pneumonie behandelten Patienten sollten zusätzlich zu den Respirationstraktmaterialien Blutkulturen abgenommen werden, da der Nachweis ätiologisch bedeutsamer Pneumonieerreger wie z.B. Streptococcus pneumoniae in manchen Fällen nur aus Blutkulturen und nicht aus dem Respirationstrakt gelingt. Die Abnahme der Blutkulturen sollte ebenfalls möglichst vor der Einleitung einer antibiotischen Therapie erfolgen, wobei die Gewinnung zweier Blutkulturen im Abstand von 1 Stunde zu empfehlen ist, um die Sensitivität der Untersuchung zu erhöhen.

Serologische Untersuchungen kommen vor allem bei der Diagnostik der atypischen Pneumonieerreger zum Einsatz. Dabei ist zu bedenken, dass die serologische Diagnostik in vielen Fällen in den ersten Tagen der akuten Symptomatik noch negativ ausfällt. Insbesondere die Ausbildung von Antikörpern gegen Chlamydia pneumoniae und Legionellen kann Wochen dauern. Auf jeden Fall sollte jedoch in der Akutphase Serum des Patienten gewonnen und untersucht werden, damit bei einer zweiten Untersuchung in der Rekonvaleszenzphase ein Vergleichsserum zur Verfügung steht und somit über einen nachgewiesen Titeranstieg die Diagnose gesichert werden kann. Zur Diagnose einer Legionellenpneumonie steht neben den kulturellen Anzuchtverfahren aus dem Respirationstrakt, die leider keine hohe Sensitivität aufweisen, und dem serologischen Nachweis der Antikörperausbildung mit dem Antigennachweis im Urin ein einfacher und schneller Test zur Verfügung, der eine gute Sensitivität und Spezifität aufweist und auch noch einige Tage nach Beginn der Antibiose positiv ausfällt. In zunehmendem Maße werden in Zukunft zur Diagnostik atypischer Pneumonien Nukleinsäure-Amplifikationstechniken angewandt werden, die erregerspezifische Nukleotidsequenzen nachweisen. Viele dieser 
Verfahren sind bisher jedoch noch nicht ausreichend standardisiert und werden erst in den nächsten Jahren routinemäßig zur Verfügung stehen.

In zahlreichen Studien wurde gezeigt, dass trotz umfangreicher diagnostischer Maßnahmen bei klinisch eindeutigen Symptomen in bis zu 50\% der Fälle kein Erregernachweis gelingt. Da sich das Spektrum der Erreger, die als Auslöser einer Pneumonie in Betracht gezogen werden müssen, in den letzten 30 Jahren um Erreger wie Moraxella catarrhalis, Legionellen und Chlamydien erweitert hat, bleibt abzuwarten, ob in Zukunft neue, bisher nicht als Pneumonieerreger bekannte Mikroorganismen entdeckt werden oder es durch verbesserte diagnostische Maßnahmen gelingt, die Ätiologie dieser bisher ungeklärten Fälle aufzudecken.

Diese Situation sollte aber nicht zu dem Schluss verleiten, keine ätiologische Diagnose anzustreben und die entsprechenden klinischen Fälle ausschließlich einer empirischen Therapie zuzuführen. Die Isolierung und Resistenztestung der Mikroorganismen ist wichtig, um die Therapie des einzelnen Patienten anhand des Kulturergebnisses zu optimieren und bei Vorliegen eines resistenten Erregers gezielt umzustellen. Dies ermöglicht es, allgemeine Trends in der Resistenzentwicklung einzelner Mikroorganismen $\mathrm{zu}$ erfassen und daraufhin ggf. die Empfehlungen zur empirischen Therapie anzupassen sowie einen ungezielten Antibiotikaeinsatz, der der Entwicklung von Resistenzen Vorschub leistet, zu verhindern.

\section{Keimspektrum}

$\mathrm{Zu}$ den am häufigsten isolierten Keimen bei einer bakteriellen, ambulant erworbenen Pneumonie gehören Streptococcus pneumoniae, Mycoplasma pneumoniae, Haemophilus influenzae und in Untersuchungen der letzten Jahre Chlamydia pneumoniae, Staphylococcus aureus, Klebsiellen und Moraxella catarrhalis. Legionellen und Coxiellen kommen ebenfalls als Erreger in Betracht, sind aber in den meisten Studien bei weniger als 5\% der Patienten als Ursache einer ambulant erworbenen Pneumonie zu finden (s. Tab. 2). Unter den viralen Erregern haben die Influenzaviren vor allem bei älteren Patienten die größte Bedeutung. Bei ca. $40 \%$ dieser Patienten kommt es im Rahmen einer bakteriellen Superinfektion im weiteren Verlauf zur Ausbildung pulmonaler Infiltrate und damit $\mathrm{zu}$ einer therapiebedürftigen bakteriellen Infektion der Lunge. RS-Viren und Adenoviren spielen vor allem im Kindesalter eine Rolle als Pneumonieerreger. Bei immunsupprimierten $\mathrm{Pa}$ tienten sind eine Reihe von zusätzlichen Pneumonieerregern in Betracht zu ziehen, neben Schimmelpilzen und Pneumocystis carinii spielen Cytomegalieviren eine wichtige Rolle.
Tab. 2 Erregerspektrum ambulant erworbener Pneumonien in europäischen Studien

\begin{tabular}{lc}
\hline Erreger & Prozentualer Anteil \\
\hline Streptococcus pneumoniae & $15-46$ \\
Mycoplasma pneumoniae & $4-10$ \\
Legionella spp. & $4-7$ \\
Haemophilus influenzae & $2-6$ \\
Chlamydia pneumoniae & bis zu $11^{\text {a }}$ \\
Klebsiella spp. & $1-2$ \\
Staphylococcus aureus & $1-2$ \\
Moraxella catarrhalis & $0-1$ \\
Andere bakterielle Erreger & $2-4$ \\
Virale Ätiologie & $4-12$ \\
Ätiologie ungeklärt & $32-45$ \\
\hline
\end{tabular}

Modifiziert nach $(7,26,40)$

${ }^{\mathrm{a}} \mathrm{C}$. pneumoniae ist in den älteren Studien als Pneumonieerreger noch nicht erfasst, die hier angegebenen Zahlen stützen sich daher auf eine neuere Untersuchung (47)

\section{Therapie}

Die empirische Therapie eines Patienten der Intensivstation mit ambulant erworbener Pneumonie sollte in jedem Fall neben den bakteriellen Erregern einer typischen Lobärpneumonie wie S. pneumoniae die Erreger einer atypischen Pneumonie mit einschließen. Die aktuellen Empfehlungen der IDSA (Infectious Disease Society of America) (2) aus dem Jahre 2000 sehen für Patienten, die aufgrund einer ambulant erworbenen Pneumonie eine intensivmedizinsche Behandlung brauchen, eine Kombination eines Beta-Laktam-Antibiotikums wie z.B. Drittgenerations-Cephalosporins wie Cefotaxim oder Ceftriaxon mit einem Macrolid oder einem Fluorochinolon vor. Diese Empfehlungen beruhen auf mehreren aktuellen Studien, die eine reduzierte Mortalität und eine kürzere Aufenthaltsdauer für Patienten nachweisen konnten, die diese Kombinationstherapie erhielten $(20,23,46)$. Der therapeutische Erfolg der Kombinationstherapie liegt möglicherweise darin begründet, dass die zwei häufigsten Erreger einer letal verlaufenden Pneumonie, S. pneumoniae und L. pneumophila, durch dieses Regime optimal erfasst werden.

\section{Nosokomiale Pneumonie und beatmungsassoziierte Pneumonie}

Nach einer Beatmungsdauer von mehr als zwei Tagen kann sich eine Pneumonie als Komplikation eines Traumas oder eines chirurgischen Eingriffs entwickeln. Diese beatmungsassoziierte Pneumonie („Ventilator associated pneumonia“ oder VAP) betrifft etwa $12 \%$ der Patienten nach ausgedehntem Trauma (1) und kann ebenfalls ein ARDS verursachen. Die VAP ist mit 38\% eine von zwei Hauptursachen (Lun- 
genkontusion 42\%) des „späten“ (nach mehr als $48 \mathrm{~h}$ ) posttraumatischen ARDS (15).

Bei Patienten mit primärem ARDS ohne initialen Erregernachweis findet sich im Verlauf der Beatmung in $30-50 \%$ der Fälle ein positiver mikrobiologischer Befund. Dieser ist aber in $60 \%$ der Fälle trotz bilateraler Infiltrate einseitig $(15,37)$ und korreliert schlecht mit den klinischen Zeichen einer Pneumonie (49). Bei den wenigen Patienten, bei denen der Kulturbefund beidseitig positiv ist, sind meist mehrere Mikroorganismen beteiligt (37). Im Übrigen handelt es sich in allen Untersuchungen jedoch um das typische Keimspektrum, an dessen Spitze Pseudomonas aeruginosa und Staphylococcus aureus stehen. Papazian et al. weisen darauf hin, dass bei negativem Kulturbefund die Lungenbiopsie bei 18 von 36 Patienten eine bis dahin nicht entdeckte CMV-Infektion ergab. Allerdings beeinflusste bei diesen Patienten eine spezifische Therapie die Letalität des ARDS von allgemein rund 50\% nicht (42).

\section{Mikrobiologische Diagnostik}

Auf die verschiedenen Techniken zur Gewinnung von Probenmaterialien aus dem Respirationstrakt wurde bereits im vorherigen Abschnitt eingegangen. Die regelmäßige Abnahme von Materialien aus dem Respirationstrakt bei Intensivpatienten als mikrobiologisches Monitoring wird von einigen Autoren abgelehnt mit dem Argument, dass dieses Vorgehen nicht kosteneffizient sei. Dieses Monitoring erlaubt es jedoch, die Besiedlung mit potentiellen Pneumonieerregern frühzeitig zu erfassen und ermöglicht es bei Auftreten von Infektionszeichen, diese in der antibiotischen Therapie zu erfassen. Ein signifikanter Anteil der Erreger nosokomialer Pneumonien hat zuvor zu einer Besiedlung des oberen Respirationstraktes und des Gastrointestinaltraktes geführt und verursacht über eine endogene Infektion die Pneumonie. In knapp $70 \%$ der Fälle geht eine Kolonisation des Respirationstraktes der Entwicklung einer Pneumonie voraus (18). $\mathrm{Ob}$ allerdings die regelmäßige Durchführung von Rachen- und Analabstrichen, wie sie von einigen Autoren zur Überwachung des Besiedlungsstatus empfohlen wird (51), eine sinnvolle Routinemaßnahme ist, ist nicht abschließend geklärt. Da der Erfolg einer Pneumonietherapie ganz wesentlich davon abhängig ist, ob die initial eingeleitete Antibiose den Erreger erfasst (30), liefert die Kenntnis des Besiedlungsstatus allerdings einen Vorteil für die optimale antibiotische Behandlung endogen erworbener Pneumonien.

\section{Keimspektrum}

Das Keimspektrum der nosokomialen Pneumonien beinhaltet neben exogenen Erregern, die durch das Krankenhauspersonal auf den Patienten übertragen
Tab. 3 Bakterielles Erregerspektrum nosokomial erworbener Pneumonien in europäischen Studien

\begin{tabular}{|c|c|}
\hline Erreger & Prozentualer Anteil \\
\hline Pseudomonas aeruginosa & 19-31 \\
\hline Staphylococcus aureus & $15-33$ \\
\hline Enterobacteriaceae & $5-31$ \\
\hline Legionella spp. & $2-10$ \\
\hline Haemophilus influenzae ${ }^{a}$ & $5-10$ \\
\hline Streptococcus pneumoniae ${ }^{a}$ & $5-18$ \\
\hline
\end{tabular}

Modifiziert nach $(5,14)$

${ }^{a}$ Nachweis vor allem bei nosokomialen Pneumonien innerhalb der ersten Woche des stationären Aufenthaltes

werden, vor allem endogene Bakterien, die den oropharyngealen Bereich des Patienten besiedelt haben und durch Mikroaspiration in die tieferen Atemwege gelangen. Bezüglich der zu erwartenden Keime stehen bei nosokomialen und beatmungsassoziierten Pneumonien gram-negative Erreger (vor allem Pseudomonas aeruginosa) im Vordergrund (s. Tab. 3), aber auch gram-positivie Erreger wie Staphylococcus aureus sind in Betracht $\mathrm{zu}$ ziehen. In Abhängigkeit von der lokalen Situation einer Klinik sind Legionellen als Erreger zu erwarten, und es wird sich bei einem Teil der $S$. aureus-Isolate um Methicillin-resistente Stämme (MRSA) handeln.

\section{Therapie}

Die antibiotische Behandlung nosokomial erworbener Pneumonien ist darauf ausgerichtet, vor allem ein breites Spektrum gram-negativer Enterobacteriaceae sowie Pseudomonas aeruginosa zu erfassen. Zur empirischen Antibiose wird daher bei beatmeten Patienten ein Carbapenem (Meropenem oder Imipenem), oder eine Kombination eines $P$. aeruginosa erfassenden $\mathrm{Pe}-$ nicillins oder Cephalosporins (z.B. Piperacillin oder Ceftazidim) mit einem P. aeruginosa wirksamen Aminoglykosid (Tobramycin oder Amikacin) empfohlen. Ist die lokale Wasserversorgung einer Klinik mit Legionellen belastet, so ist eine zusätzliche Gabe von Erythromycin oder Azithromycin indiziert. Vor allem bei neutropenischen Patienten mit $\leq 500$ Leukozyten/ $\mathrm{mm}^{3}$ sind Pilze, in erster Linie Candida. spp. und Schimmelpilze, als Erreger in Betracht zu ziehen. Eine empirische Gabe von Amphotericin B bei diesen $\mathrm{Pa}-$ tienten ist angezeigt, wenn nach einer einwöchigen Antibiose mit einem Breitspektrumantibiotikum keine Entfieberung eingetreten ist (28). Die Wirksamkeit einer empirischen Fluconazoltherapie konnte bisher nicht belegt werden (28).

Eine Methode, die zur Vermeidung nosokomialer Pneumonien erstmals in den 80iger Jahren angewandt wurde (48), ist die selektive Darm-Dekontamination (SDD). Durch eine topische, systemische, 
oder kombiniert topisch und systemische Antibiose soll die Besiedlung mit Erregern, die eine nosokomiale Pneumonie hervorrufen können, vermieden werden und so die Rate der beatmungsassoziierten Pneumonien gesenkt werden. Während in Studien verschiedener Arbeitsgruppen und in mehreren Meta-Analysen eine Senkung der Rate nosokomialer Pneumonien gezeigt werden konnte, waren die Effekte auf das Überleben der Patienten, eine Reduzierung der Beatmungsdauer, oder die Dauer des Aufenthaltes auf der Intensivstation nicht in ähnlich überzeugender Weise nachweisbar. Ein kombiniertes Regime aus topischer plus systemischer Antibiose scheint in der Prophylaxe der Pneumonien am effektivsten zu sein. Insgesamt überwiegt jedoch bei vielen Experten die Sorge, aufgrund der eingesetzten Antibiose resistente Erreger $\mathrm{zu}$ selektionieren. Es konnte gezeigt werden, dass SDD zu einer Verschiebung der lokalen Mikroflora führt und die Besiedlung mit Stämmen, die gegen die eingesetzten Antibiotika resistent sind, begünstigt (52). Ein massiver Anstieg der MRSA-Isolate, der unter SDD-Regimes wiederholt beobachtete wurde, ist in diesem Zusammenhang sicher eine der größten Gefahren $(29,33)$. In Abwägung der vorliegenden Daten wird zur Zeit der routinemäßige Einsatz der Methode sehr kontrovers diskutiert (9). Zu bedenken ist vor allem, dass in den Publikationen, die den Einsatz befürworten, die Resistenzentwicklung nicht in die Entscheidungsfindung mit einbezogen wurde (32).

\section{ARDS als Folge der Sepsis}

Mit über der Hälfte der Fälle sind Sepsis und SIRS („Systemic Inflammatory Response Syndrome“) häufigste Ursache des ARDS (10). Von den meisten Autoren wird allerdings nicht nach der primären Ursache der Sepsis differenziert, so dass diese Zahl in der Regel die Patienten mit sekundär septisch verlaufenden Pneumonien einschließt.

Das Multiorganversagen als Folge einer Sepsis ist mit rund $50 \%$ die häufigste Todesursache bei Patienten mit ARDS und gleichzeitig ein wichtiger Prädiktor (neben einem Alter $>65$ Jahre und Begleiterkrankungen wie Immunsuppression durch HIV, Tumorleiden, Organtransplantation oder chronische Steroidtherapie) für die Letalität des $\operatorname{ARDS}(21,56)$. Für die mikrobiologische Diagnostik und Therapie des ARDS als Sepsisfolge muss daher vor allem die primäre Ursache der Sepsis berücksichtigt werden. Dieser Ansatz wird unterstützt durch eine Untersuchung von Davidson und Mitarbeitern: In einem Vergleich von 127 Patienten mit ARDS mit 127 „matched controls“ ohne ARDS (gepaart für Alter, Begleiterkrankungen und Häufigkeit von Trauma und Sepsis) war die Letalität in beiden Gruppen gleich, d.h. andere Erkrankungen hatten prognostisch wesentlich größere Bedeutung als das ARDS (17).

\section{Diagnostik}

Die Prognose dieser Patienten wird entscheidend durch die Sepsis bestimmt (17) und ist daher vor allem von der erfolgreichen antibiotischen Behandlung der Sepsis abhängig. Die Abnahme von Blutkulturen möglichst im Fieberanstieg ist weiterhin, trotz geringer Sensitivität dieser Untersuchung, der Goldstandard der Sepsisdiagnostik. Um die Sensititvität der Diagnostik zu erhöhen, sind bis zu drei Abnahmen pro Tag sind sinnvoll, wobei in der Regel ein Mindestabstand von einer Stunde zwischen zwei Abnahmen eingehalten werden sollte und jeweils eine aerobe und eine anaerobe Kultur des abgenommen Materials zu empfehlen ist. Da das Belüften aerober Blutkulturflaschen eine der häufigsten Kontaminationsquellen ist (44), sollte es ausschließlich im mikrobiologischen Labor unter kontrollierten Bedingungen erfolgen (mit gestopften Kanülen unter der Sicherheitswerkbank). Weiterhin zu beachten ist, dass ein Teil der neueren Blutkulturflaschen ein Gasgemisch enthält, das eine Belüftung überflüssig macht und diese Kontaminationsquelle somit eliminiert werden kann.

$\mathrm{Da}$ auch bei der Sepsis die mikrobiologische Diagnostik ganz wesentlich durch eine gleichzeitige Gabe von Antibiotika beeinträchtigt wird (16), ist immer eine Abnahme vor Einleitung einer empirischen Antibiose anzustreben. Falls der Patient bereits eine Antibiose erhält, ist in Abhängigkeit von der klinischen Situation ein Absetzen der Therapie $\mathrm{zu}$ diagnostischen Zwecken zu erwägen. Einige Blutkulturmedien enthalten Zusätze, die im Blut vorhandene Antibiotika absorbieren und auf diese Weise eine erfolgreiche Diagnostik auch unter antibiotischer Therapie ermöglichen sollen. Die Sensitivität dieser Kulturmedien ist gegenüber herkömmlichen Medien verbessert worden, liegt jedoch nicht in den Bereichen, die ohne Antibiotika erzielt werden (53, 55). Ein Einsatz dieser Kulturmedien sollte auf jeden Fall bei den Patienten erfolgen, deren klinische Situation ein Absetzen der antibiotischen Therapie nicht erlaubt. Für die gesicherte Identifikation eines Sepsiserregers wird in der Regel der zweimalige Nachweis desselben Keims in zwei zu unterschiedlichen Zeitpunkten entnommenen Blutkulturen gefordert. Die routinemäßige Anlage von Blutkulturen ohne Hinweise auf eine Infektion als sog. „Surveillance" ist abzulehnen, da keine Vorteile für den $\mathrm{Pa}$ tienten nachzuweisen sind und die Rate falsch positiver Kulturen durch Kontaminationen deutlich ansteigt (31). 
Um eine eventuell vorliegende bakterielle Besiedlung eines zentralvenösen Zugangs nicht mit einer Sepsis $\mathrm{zu}$ verwechseln, ist prinzipiell eine Venenpunktion zur Materialgewinnung zu empfehlen. Um die Fragestellung „Katheterbesiedlung oder Sepsis“ zu klären, kann auch eine parallele Blutentnahme aus dem zentralvenösen Katheter und durch Venenpunktion sinnvoll sein. Liegt der Verdacht eines infizierten Katheters als Ausgangspunkt der Sepsis vor, so sollte nach Entfernen des Katheters eine semiquantitative Kultur des entfernten Katheters erfolgen, um die Verdachtsdiagnose $\mathrm{zu}$ verifizieren. Die mikrobiologische Diagnostik der Sepsis sollte neben den Blutkulturen die Erfassung des Ausgangsherdes der Infektion beinhalten, um eine Sanierung dieses Fokus zu erlauben. Die wichtigsten Ausgangsherde sind das Urogenitalsystem, intravasale Katheter, der Respirationstrakt sowie Haut und Weichteile (54). Für eine umfassende Darstellung der zu diesem Zweck indizierten mikrobiologischen Diagnostik wird auf die einschlägige Literatur verwiesen.

\section{Keimspektrum}

Das Keimspektrum der zu erwartenden Erreger einer Sepsis ist von der Grunderkrankung des Patienten abhängig. Dabei konnten in den letzten Jahren in Europa ein Anstieg der gram-positiven Erreger und Pilze und eine Abnahme der gram-negativen Erreger beobachtet werden. Häufig isolierte bakterielle Erreger der Sepsis sind in Tabelle 4 aufgeführt.

\section{Therapie}

Die Prognose eines Patienten mit Sepsis wird entscheidend davon beeinflusst, ob die eingeleitete Antibiose den Erreger adäquat erfasst. Dabei ist die Kenntnis des Erregers und der Antibiotika-Empfindlichkeit wichtig, um eine gezielte Antibiose einzuleiten, die in vielen Fällen zu einer Senkung der Letalität beiträgt (54). Bevor eine Kenntnis des Erregers vorliegt oder bei trotz eindeutiger klinischer Symptomatik negati-

Tab. 4 Erregerspektrum der Blutkulturisolate bei Sepsis

\begin{tabular}{lc}
\hline Erreger & Prozentualer Anteil \\
\hline Staphylococcus aureus & $17-20$ \\
Koagulase-neg. Staphylokokken & $9-24$ \\
Enterokokken & $6-8$ \\
Streptokokken & $4-14$ \\
Escherichia coli & $11-24$ \\
Enterobacter spp. & $4-5$ \\
Klebsiella spp. & $4-5$ \\
Pseudomonas aeruginosa & 4 \\
Candida spp. & $1-4$ \\
\hline
\end{tabular}

Modifiziert nach $(22,43,50)$ ven Blutkulturen, ist die Kombination eines Betalactam-Antibiotikums mit einem Aminoglykosid aufgrund der synergistischen Wirkung dieser Kombination noch immer die erste Wahl in der kalkulierten Antibiotikatherapie des septischen Patienten. Dabei bestimmt vor allem die Auswahl des Betalaktam-Antibiotikums, ob eine gute Staphylokokkenwirksamkeit erreicht wird oder der Schwerpunkt auf der Erfassung resistenter gram-negativer Erreger, wie z.B. Pseudomonas spp. liegt. Diese Auswahl wird in der Regel durch die Grunderkrankung des Patienten und den vermuteten Ausgangsherd sowie die Länge des stationären Aufenthaltes (nosokomiale Erreger) und die lokale Resistenzsituation der häufig isolierten Erreger beeinflusst. Nach Vorliegen des Antibiogramms muss überprüft werden, ob die initial gewählte Kombination eine optimale Erfassung sicherstellt oder ob eine Anpassung der Antibiose nötig ist. Für die detaillierte Diskussion einzelner Therapieschemata und deren Vor- und Nachteile sei auf mehrere ausführliche Übersichtsartikel zu diesem Thema verwiesen $(8,19)$.

\section{Posttraumatisches ARDS}

Ein ausgedehntes Trauma kann - auch wenn es nicht durch eine pulmonale Infektion kompliziert ist über mehrere Mechanismen ein akutes Lungenversagen verursachen: Wichtigster Faktor ist offenbar die systemische Entzündungsreaktion mit Leukozytenaktivierung und Störung der Kapillarpermeabilität (3). Auch die Transfusion großer Mengen Blut führt vermutlich über diesen Mechanismus zur Lungenschädigung. Daneben spielt zwar das direkte Thoraxtrauma eine Rolle; in einer Untersuchung von Charash und Mitarbeitern hatte aber der Zeitpunkt, zu dem eine Femurfraktur bei mehrfach traumatisierten Patienten versorgt wurde, größeren Einfluss auf die Häufigkeit pulmonaler Komplikationen (12\% bei früher gegen $45 \%$ bei später Versorgung) als ein zusätzlich vorhandenes Thoraxtrauma (11).

In diese Gruppe kann man auch diejenigen Patienten einordnen, bei denen sich nach einem größeren chirurgischen oder herzchirurgischen Eingriff ein ARDS ohne pulmonale Infektion entwickelt. Die Häufigkeit der respiratorischen Insuffizienz nach Eingriffen mit extrakorporaler Zirkulation (EKZ) hängt von der Größe des Eingriffs sowie der Dauer der EKZ ab und wird daher sehr unterschiedlich angegeben. Das eigentliche ARDS ist mit etwa $1,5 \%$ der Patienten aber selten (38).

\section{Diagnostik}

Die mikrobiologische Diagnostik bei Patienten mit ARDS nach Trauma strebt die Abgrenzung einer Pneumonie von einem reaktiv durch Entzündungs- 
mediatoren vermittelten ARDS an. Leider ist es bisher nicht gelungen, einen einzelnen Laborparameter oder ein diagnostisches Schema zu identifizieren, das eine sichere Unterscheidung einer Pneumonie von einer nicht infektiologisch bedingten Entzündungsreaktion erlaubt. In dieser Situation kann das Vorliegen von $\geq 10^{4}$ Keimen $/ \mathrm{ml}$ in einer quantitativ ausgewerteten BAL diagnostisch wegweisend sein. Insgesamt muss sich die Diagnose jedoch auf eine erfahrungsbasierte Gesamtbewertung der mikrobiologischen Ergebnisse einer Pneumoniediagnostik, der Laborparameter und des klinischen Bildes stützen.

\section{Antibiotische Therapie}

Da die Abgrenzung einer Pneumonie von nicht infektiologischen Ursachen des ARDS eine schwierige klinische Diagnose darstellt, die in vielen Fällen erst post mortem gesichert werden kann, wird ein nennenswerter Anteil der Patienten unter dem Verdacht einer nosokomial erworbenen Pneumonie antibiotisch behandelt werden. $\mathrm{Zu}$ bedenken ist auch, dass durch die vorliegende Gewebeschädigung der Lunge die Entwicklung einer nosokomialen Pneumonie begünstigt wird (s.o.), so dass in vielen dieser Situationen die Indikation zur Antibiotikatherapie großzügig gestellt werden muss. In einer prospektiven Studie mit 56 ARDS-Patienten entwickelten 31 dieser Patienten (55\%) in Laufe der Erkrankung eine Pneumonie (12). Empfehlungen zu einer generellen antibiotischen Prophylaxe bei ARDS-Patienten, die weder laborchemische oder klinische Infektzeichen, noch einen relevanten Keimnachweis aus dem Respirationstrakt aufweisen, werden sehr kontrovers beur- teilt (34), da der ungezielte, langdauernde Einsatz von Antibiotika die Ausbreitung resistenter Erreger wie $P$. aeruginosa, Acinetobacter sp. und Stenotrophomonas sp. begünstigt.

\section{Fazit}

Wegen der Schwere des Krankheitsbildes und der meist vorhandenen Infektionszeichen ist ein Verzicht auf antibiotische Therapie in der Behandlung des ARDS nur in speziellen Einzelfällen gerechtfertigt. Die diagnostischen Probleme bei der Identifizierung eines relevanten Erregers bedingen jedoch weiterhin, dass diese Therapie meist empirisch begonnen werden muss. Da der Zeitbedarf für die Gewinnung mikrobiologischer Proben jedoch gering und diese bei Nachweis eines Pneumonieerregers von therapeutischer Relevanz ist, kann (und sollte) diese immer vor Beginn der Behandlung durchgeführt werden, ohne den Patienten zu gefährden. Bei der Planung der Therapie müssen die entsprechend der Genese des Lungenversagens relevanten Erreger möglichst vollständig erfasst und über einen ausreichenden Zeitraum in adäquater Dosierung behandelt werden. Dafür ist die genaue Kenntnis von Anamnese und klinischem Verlauf entscheidend. Es muss aber bedacht werden, dass für die Prognose und damit auch für die Therapie der Patienten Sepsis, Trauma und eventuelle Vorerkrankungen zumindest ebenso große Bedeutung haben wie das ARDS selbst. Empfehlungen für die initiale antibiotische Behandlung der $\mathrm{Pa}$ tienten sind in Tabelle 5 zusammengestellt.
Tab. 5 Empfehlungen für die initiale kalkulierte Antibiose bei Patienten mit ARDS

\begin{tabular}{|c|c|c|c|c|}
\hline Genese des ARDS & $\begin{array}{l}\text { Ambulant erworbene } \\
\text { Pneumonie (CAP) }\end{array}$ & $\begin{array}{l}\text { Beatmungsassoziierte } \\
\text { Pneumonie (VAP) }\end{array}$ & Sepsis & Trauma \\
\hline Häufige Erreger & $\begin{array}{l}\text { Streptococcus } \\
\text { pneumoniae } \\
\text { Mycoplasma } \\
\text { pneumoniae } \\
\text { Chlamydia } \\
\text { pneumoniae }\end{array}$ & $\begin{array}{l}\text { Pseudomonas } \\
\text { aeruginosa } \\
\text { Staphylococcus } \\
\text { aureus } \\
\text { Enterobacter } \\
\text { spp. }\end{array}$ & $\begin{array}{l}\text { Staphylococc. } \\
\text { aureus } \\
\text { Escherichia } \\
\text { Coli } \\
\text { Koagulase- } \\
\text { neg. Staph. }\end{array}$ & $\begin{array}{l}\text { Häufig nicht } \\
\text { identifiziert }\end{array}$ \\
\hline Initiale Therapie ${ }^{a}$ & $\begin{array}{l}\text { Ill.-Generation- } \\
\text { Cephalosporin } \\
\text { plus } \\
\text { Makrolid oder } \\
\text { Fluorchinolon }\end{array}$ & $\begin{array}{l}\text { Carbapenem } \\
\text { oder } \\
\text { Betalaktam plus } \\
\text { Aminoglykosid }\end{array}$ & $\begin{array}{l}\text { Betalaktam } \\
\text { plus } \\
\text { Aminoglykosid }\end{array}$ & $\begin{array}{l}\text { Bei klinischem } \\
\text { Anhalt für } \\
\text { Infektion: } \\
\Rightarrow \text { CAP } \\
\Rightarrow \text { VAP }\end{array}$ \\
\hline Beispiel & $\begin{array}{l}\text { Cefotaxim } \\
\text { plus } \\
\text { Erythromycin } \\
\text { oder Ciprofloxacin }\end{array}$ & $\begin{array}{l}\text { Imipenem } \\
\text { oder } \\
\text { Ceftazidim plus } \\
\text { Tobramycin }\end{array}$ & $\begin{array}{l}\text { Piperacillin/ } \\
\text { Tazobactam } \\
\text { plus } \\
\text { Tobramycin }\end{array}$ & s. links \\
\hline
\end{tabular}

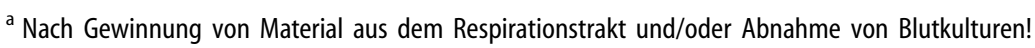




\section{Literatur}

1. Antonelli M, Moro ML, Capelli O, De Blasi RA, D’Errico RR, Conti G, Bufi M, Gasparetto A (1994) Risk factors for early onset pneumonia in trauma patients. Chest 105(1):224-228

2. Bartlett JG, Dowell SF, Mandell LA, File TM Jr, Musher DM, Fine MJ (2000) Practice guidelines for the management of community-acquired pneumonia in adults. Infectious Diseases Society of America. Clin Infect Dis 31(2):347-382

3. Bauer TT, Monton C, Torres A, Cabello $\mathrm{H}$, Fillela X, Maldonado A, Nicolas JM, Zavala E (2000) Comparison of systemic cytokine levels in patients with acute respiratory distress syndrome, severe pneumonia, and controls. Thorax 55(1):46-52

4. Baughman RP (2000) Protected-specimen brush technique in the diagnosis of ventilator- associated pneumonia. Chest 117(4 Suppl 2):203-206

5. Bergogne-Berezin E (1995) Treatment and prevention of nosocomial pneumonia. Chest 108(2 Suppl):26-34

6. Bernard GR, Artigas A, Brigham KL, Carlet J, Falke K, Hudson L, Lamy M, Legall JR, Morris A, Spragg R (1994) The American-European Consensus Conference on ARDS. Definitions, mechanisms, relevant outcomes, and clinical trial coordination. Am J Respir Crit Care Med 149(3 Pt 1):818824

7. Blanquer $\mathrm{J}$, Blanquer $\mathrm{R}$, Borras $\mathrm{R}$, Nauffal D, Morales P, Menendez R, Subias I, Herrero L, Redon J, Pascual J (1991) Aetiology of community acquired pneumonia in Valencia, Spain: a multicentre prospective study. Thorax 46(7):508-511

8. Bodman K, Vogel F (2001) Antimikrobielle Therapie der Sepsis. Chemotherapie-Journal 10:43-54

9. Bonten MJ, Kullberg BJ, van Dalen R, Girbes AR, Hoepelman IM, Hustinx W, van der Meer JW, Speelman P, Stobberingh EE, Verbrugh HA, Verhoef J, Zwaveling JH (2000) Selective digestive decontamination in patients in intensive care. The Dutch Working Group on Antibiotic Policy. J Antimicrob Chemother 46(3):351-362

10. Brunkhorst FM, Eberhard OK, Brunkhorst R (1999) Discrimination of infectious and noninfectious causes of early acute respiratory distress syndrome by procalcitonin. Crit Care Med 27(10):2172-2176

11. Charash WE, Fabian TC, Croce MA (1994) Delayed surgical fixation of femur fractures is a risk factor for pulmonary failure independent of thoracic trauma. J Trauma 37(4):667672
12. Chastre J, Trouillet JL, Vuagnat A, Joly-Guillou ML, Clavier H, Dombret MC, Gibert C (1998) Nosocomial pneumonia in patients with acute respiratory distress syndrome. Am J Respir Crit Care Med 157(4 Pt 1): 1165-1172

13. Confalonieri $\mathrm{M}$, Potena A, Carbone G, Porta R, Tolley E, Umberto Meduri G (1999) Acute respiratory failure in patients with severe community-acquired pneumonia. A prospective randomized evaluation of noninvasive ventilation. Am J Respir Crit Care Med 160:1585-1591

14. Craven DE, Steger KA (1995) Epidemiology of nosocomial pneumonia. New perspectives on an old disease. Chest 108(2 Suppl):1-16

15. Croce MA (2000) Diagnosis of acute respiratory distress syndrome and differentiation from ventilator-associated pneumonia. Am J Surg 179 (2 Suppl 1):26-29

16. Darby JM, Linden P, Pasculle W, Saul M (1997) Utilization and diagnostic yield of blood cultures in a surgical intensive care unit. Crit Care Med 25(6):989-994

17. Davidson TA, Rubenfeld GD, Caldwell ES, Hudson LD, Steinberg KP (1999) The effect of acute respiratory distress syndrome on long-term survival. Am J Respir Crit Care Med 160(6):1838-1842

18. Delclaux C, Roupie E, Blot F, Brochard L, Lemaire F, Brun-Buisson C (1997) Lower respiratory tract colonization and infection during severe acute respiratory distress syndrome: incidence and diagnosis. Am J Respir Crit Care Med 156(4 Pt 1):1092-1098

19. Dellinger RP (1999) Current therapy for sepsis. Infect Dis Clin North Am 13(2):495-509

20. Dudas V, Hopefl A, Jacobs R, Guglielmo BJ (2000) Antimicrobial selection for hospitalized patients with presumed community-acquired pneumonia: a survey of nonteaching US community hospitals. Ann Pharmacother 34(4):446-452

21. Ferring M, Vincent JL (1997) Is outcome from ARDS related to the severity of respiratory failure? Eur Respir J 10(6):1297-1300

22. Geerdes HF, Ziegler D, Lode H, Hund M, Loehr A, Fangmann W, Wagner J (1992) Septicemia in 980 patients at a university hospital in Berlin: prospective studies during 4 selected years between 1979 and 1989. Clin Infect Dis 15(6):991-1002
23. Gleason PP, Meehan TP, Fine JM, Galusha DH, Fine MJ (1999) Associations between initial antimicrobial therapy and medical outcomes for hospitalized elderly patients with pneumonia. Arch Intern Med 159(21):2562-2572

24. Headley AS, Tolley E, Meduri GU (1997) Infections and the inflammatory response in acute respiratory distress syndrome. Chest 111(5):1306-1321

25. Hilbert G, Gruson D, Vargas F, Valentino R, Gbikpi-Benissan G, Dupon M, Reiffers J, Cardinaud J (2001) Noninvasive ventilation in immunosuppressed patients with pulmonary infiltrates, fever, and acute respiratory failure. N Engl J Med 344:481-487

26. Hoffken G, Steinhoff D, Lode H (1995) Ätiologie von ambulant erworbenen Pneumonien bei älteren Menschen. Chemotherapie J 4 (Suppl 7):13-19

27. Holtermann W, Kramer M, Geppert $P$, van Wickern $M$, Lukasewitz $P$ (1998) Epidemiology, clinical aspects and prognosis of severe progressive community-acquired pneumonia. Pneumologie 52(5):263-270

28. Hughes WT, Armstrong D, Bodey GP, Brown AE, Edwards JE, Feld R, Pizzo $\mathrm{P}$, Rolston KV, Shenep JL, Young LS (1997) 1997 guidelines for the use of antimicrobial agents in neutropenic patients with unexplained fever. Infectious Diseases Society of America. Clin Infect Dis 25(3):551-573

29. Kaufhold A, Behrendt W, Krauss T, van Saene H (1992) Selective decontamination of the digestive tract and methicillin- resistant Staphylococcus aureus. Lancet 339(8806):1411-1412

30. Kollef MH, Sherman G, Ward S, Fraser VJ (1999) Inadequate antimicrobial treatment of infections: a risk factor for hospital mortality among critically ill patients. Chest 115(2): 462-474

31. Levin PD, Hersch M, Rudensky B, Yinnon AM (1997) Routine surveillance blood cultures: their place in the management of critically ill patients. J Infect 35(2):125-128

32. Liberati A, D'Amico R, Pifferi S, Leonetti C, Torri V, Brazzi L, Tinazzi A (2000) Antibiotics for preventing respiratory tract infections in adults receiving intensive care. Cochrane Database Syst Rev 2

33. Lingnau W, Berger J, Javorsky F, Fille M, Allerberger F, Benzer H (1998) Changing bacterial ecology during a five-year period of selective intestinal decontamination. J Hosp Infect 39(3): 195-206 
34. Lynch JP (2001) 3rd. Hospital-acquired pneumonia: risk factors, microbiology, and treatment. Chest 119(2 Suppl):373-384

35. Marquette $\mathrm{CH}$ (2001) Diagnosis and treatment of nosocomial pneumonia: bronchial fibroscopy, protected brushing and/or bronchial lavage is not indispensable. Rev Pneumol Clin 57(2):124-131

36. Mauch H, Wagner J, Marklein G (1999) MiQ-Richtlinie 7 Infektionen der tiefen Atemwege Teil I. Urban \& Fischer

37. Meduri GU, Reddy RC, Stanley T, ElZeky F (1998) Pneumonia in acute respiratory distress syndrome. A prospective evaluation of bilateral bronchoscopic sampling. Am J Respir Crit Care Med 158(3):870-875

38. Messent M, Sullivan K, Keogh BF, Morgan CJ, Evans TW (1992) Adult respiratory distress syndrome following cardiopulmonary bypass: incidence and prediction. Anaesthesia 47(3):267-268

39. Namias N, Harvill S, Ball S, McKenney MG, Sleeman D, Ladha A, Civetta J (1998) A reappraisal of the role of Gram's stains of tracheal aspirates in guiding antibiotic selection in the surgical intensive care unit. J Trauma 44(1):102-106

40. Ortqvist A, Hedlund J, Grillner L, Jalonen E, Kallings I, Leinonen M, Kalin M (1990) Aetiology, outcome and prognostic factors in community-acquired pneumonia requiring hospitalization. Eur Respir J 3(10): 1105-1113

41. Papazian L, Autillo-Touati A, Thomas P, Bregeon F, Garbe L, Saux P, Seite R, Gouin F (1997) Diagnosis of ventilator-associated pneumonia: an evaluation of direct examination and presence of intracellular organisms. Anesthesiology 87(2):268-276
42. Papazian L, Thomas P, Bregeon F, Garbe L, Zandotti C, Saux P, Gaillat F, Drancourt M, Auffray JP, Gouin F (1998) Open-lung biopsy in patients with acute respiratory distress syndrome. Anesthesiology 88(4):935-944

43. Rosenthal E (1993) Epidemiologie von Septikämie-Erregern. Deutsch Med Wochenschr 118:1269-1275

44. Simhon A, Rahav G, Shapiro M, Block C (2001) Skin disease presenting as an outbreak of pseudobacteremia in a laboratory worker. J Clin Microbiol 39(1):392-393

45. Singh N, Falestiny MN, Rogers P, Reed MJ, Pularski J, Norris R, Yu VL (1998) Pulmonary infiltrates in the surgical ICU: prospective assessment of predictors of etiology and mortality. Chest 114(4):1129-1136

46. Stahl JE, Barza M, DesJardin J, Martin R, Eckman MH (1999) Effect of macrolides as part of initial empiric therapy on length of stay in patients hospitalized with community-acquired pneumonia. Arch Intern Med 159(21):2576-2580

47. Steinhoff D, Lode H, Ruckdeschel G, Heidrich B, Rolfs A, Fehrenbach FJ, Mauch H, Hoffken G, Wagner J (1996) Chlamydia pneumoniae as a cause of community-acquired pneumonia in hospitalized patients in Berlin. Clin Infect Dis 22(6):958-964

48. Stoutenbeek CP, van Saene HK, Miranda DR, Zandstra DF (1984) The effect of selective decontamination of the digestive tract on colonisation and infection rate in multiple trauma patients. Intensive Care Med 10(4): 185-192

49. Sutherland KR, Steinberg KP, Maunder RJ, Milberg JA, Allen DL, Hudson LD (1995) Pulmonary infection during the acute respiratory distress syndrome. Am J Respir Crit Care Med 152(2):550-556
50. Trautmann (1997) Computergestützte prospektive Erfassung nosokomialer Septikämien in einem Universitätsklinikum. Hyg Med 22:467-473

51. van Saene HK, Damjanovic V, Murray AE, de la Cal MA (1996) How to classify infections in intensive care units - the carrier state, a criterion whose time has come? J Hosp Infect 33(1):1-12

52. Vollaard EJ, Clasener HA (1994) Colonization resistance. Antimicrob Agents Chemother 38(3):409-414

53. Weinstein MP, Mirrett S, Reimer LG, Wilson ML, Smith-Elekes S, Chuard CR, Joho KL, Reller LB (1995) Controlled evaluation of BacT/Alert standard aerobic and FAN aerobic blood culture bottles for detection of bacteremia and fungemia. J Clin Microbiol 33(4):978-981

54. Weinstein MP, Reller LB, Murphy JR, Lichtenstein KA (1983) The clinical significance of positive blood cultures: a comprehensive analysis of 500 episodes of bacteremia and fungemia in adults. I. Laboratory and epidemiologic observations. Rev Infect Dis 5(1):35-53

55. Wilson ML, Weinstein MP, Mirrett $S$, Reimer LG, Feldman RJ, Chuard CR, Reller LB (1995) Controlled evaluation of BacT/alert standard anaerobic and FAN anaerobic blood culture bottles for the detection of bacteremia and fungemia. J Clin Microbiol 33(9): 2265-2270

56. Zilberberg MD, Epstein SK (1998) Acute lung injury in the medical ICU: comorbid conditions, age, etiology, and hospital outcome. Am J Respir Crit Care Med 157(4 Pt 1):1159-1164 